\title{
Quo vadis Visuelle Jurisprudenz?
}

\section{Postulat für eine Rechtsvisualisierung im Lehr- und Lernprozess}

\author{
Aiman Khalil*
}

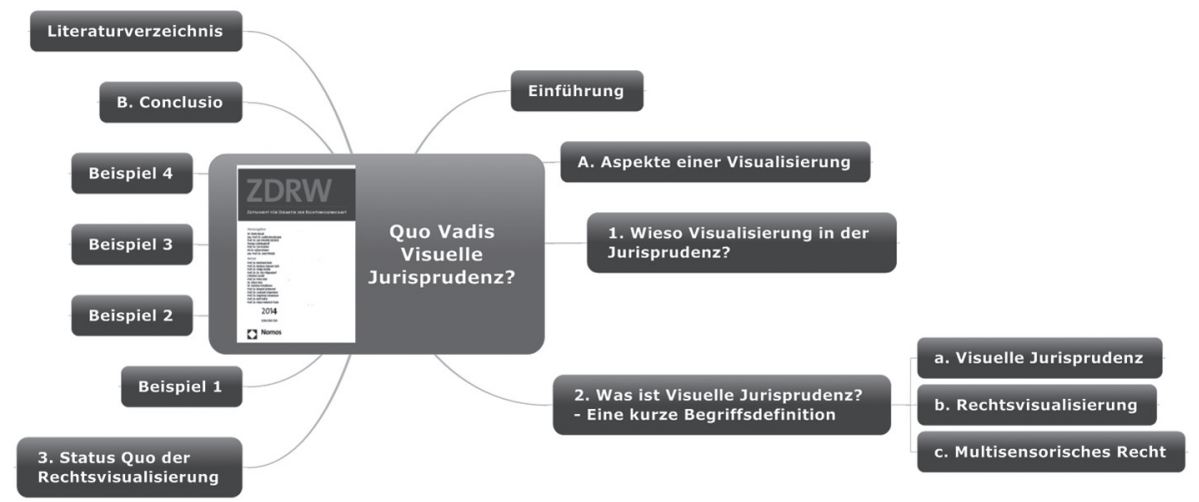

Abb. 1: Überblick Aufsatz als Mindmap von Aiman Khalil

\section{Einführung}

In den letzten fünf Jahren hat die Sensibilität für eine neue und effektivere Didaktik in der Rechtswissenschaft spürbar zugenommen. Zahlreiche lehr- und lerndidaktische Bücher sind nun auch von den wenigen, aber renommierten Rechtsverlagen in der Bundesrepublik und im deutschsprachigen Ausland initiiert und veröffentlicht worden. Viele dieser Werke werden auch rege zur basalen Studienliteratur von den Studierenden der Rechtswissenschaft, sowohl an den Universitäten, als auch an den Universities of Applied Sciences genutzt und dort speziell in den jeweiligen (nicht nur) wirtschaftsrechtlichen Bachelor-und Masterstudiengängen gelesen. ${ }^{1}$ Auch visualisierte Elemente finden vermehrt Zugang in diese Bücher. ${ }^{2}$

Dies war nicht immer so. Bereits zehn Jahre vorher begann eine Gruppe von Rechtswissenschaftlerinnen und Rechtswissenschaftlern, vornehmlich aus Europa, daran zu arbeiten, die lehr- und lerndidaktischen Herausforderungen für die Rechtswissenschaft mittels einer Rechtsvisualisierung anzugehen und in der klassischen Di-

* Aiman Khalil arbeitet im Bundesamt für zentrale Dienste und offene Vermögensfragen (BADV), einer oberen Bundesbehörde im Geschäftsbereich des Bundesministeriums der Finanzen. Die hier dargestellte Übersicht ist nicht in dienstlicher Eigenschaft verfasst und gibt ausschließlich die private Meinung des Verfassers wieder.

1 Juraspezifisch und exemplarisch für alle: Schimmel/Weinert et al., Juristische Themenarbeiten; oder Putzke, Juristische Arbeiten erfolgreich schreiben; für fachübergreifende Unterstützung findet sich exemplarisch für alle: Karmasin/Ribing, Die Gestaltung wissenschaftlicher Arbeiten; Ebster/Stalzer, Wissenschaftliches Arbeiten; Eco, Wie man eine wissenschaftliche Abschlussarbeit schreibt; wesentlich früher: Gerhards, Seminar-, Diplom und Doktorarbeit.

2 Dazu gehören übrigens auch sog. Snapshots, vgl. dazu nochmals Ebster/Stalzer, Wissenschaftliches Arbeiten, S. 60. 
daktik weitere neue Methoden für ein nachhaltiges Lernen, aber auch Lehren und darüber hinaus ${ }^{3}$ zu erforschen, zu erproben und zu etablieren. ${ }^{4}$

Der folgende Beitrag gibt einen kurzen Querschnitt über die Entwicklung einer rechtsvisualisierten Didaktik in den letzten Jahren. Gleichzeitig möchte er aufzeigen, was mit dem „pictorial turn“ bisher geleistet wurde und warum es zu keinem Stillstand kommen darf, sondern der nächste logische Schritt, eine mutige weitere Etablierung im Hinblick auf einen Visualized Turn beinhaltet. Hierbei sollen aber auch kritische Untertöne bei der bisherigen Umsetzung und auch erste, exemplarische Lösungsansätze Erwähnung finden. ${ }^{5}$

\section{A. Aspekte einer Visualisierung im Recht}

„Bilder sind der Laien Schrift und Bücher.“6 „Der Gedanke, unsere Verfassung zu bebildern, scheint für viele allzu fernliegend. "7 Diese und ähnlich klingenden Kommentare in Bezug auf die verbildlichten Aspekte der Rechtsvisualisierung sind im letzten Jahrzehnt oft in Fachgesprächen mit renommierten Lehrstuhlinhaberinnen und Lehrstuhlinhabern an rechtswissenschaftlichen Fakultäten gefallen. Diese vorsichtige Ablehnung ist einem wachsenden Interesse an einer Rechtsvisualisierung gewichen. Eine immer größer werdende Gruppe von (Rechts-) Wissenschaftlerinnen und Wissenschaftlern versucht seit einigen Jahren, die möglichen Vorteile einer Rechtsvisualisierung für den Lehr- und Lernalltag zu erforschen. Mit der Emeritierung zahlreicher Lehrstuhlinhaberinnen und Lehrstuhlinhaber sind junge und vor allem neuen und fachdisziplinübergreifenden Ideen zugeneigte wissenschaftlich orientierte Personen an den Universitäten und Hochschulen angekommen. Die Kehrseite der Medaille ist die gewichene Bereitschaft der Studierenden, zahlreiche rechtswissenschaftliche Aufsätze, Artikel und Lehrbücher sorgfältig zu prüfen und gewissenhaft zu lesen; substituiert wurde dies durch das Lesen von komprimierten Lernbüchern oder Skripten. ${ }^{8}$ Aber auch der gerade aufgezeigte vermeintliche Idealweg der letzten Jahrhunderte (das Lesen der Lehrbücher als Primärquelle) kann heute allein nicht mehr so beschritten werden. Viele Studierende sind zum einen durch die

3 Für die Praxis sei exemplarisch auf die Applikation „Normfall” von Haft verwiesen, aktueller für die visuelle Kommunikation im Strafprozess vgl. Kertai, in: MMR 2011, S. 716 ff.; international bereits für die Praxis rege tätig Haapio/Passera.

4 Z.B. Volk/Holzer et al., Panorama Strafrecht, Herzog, Strafrecht illustrated; theoretische Ansätze durch Lachmayer, in: Zeitschrift für Verkehrsrecht, 1976, S.230ff.; ders., in: Klug/Ramm et al. (Hrsg.), S. 50 ff.; Röhl/Ulbrich, Recht anschaulich - Visualisierung in der Juristenausbildung; Khalil, Visuelle Jurisprudenz; Holzer, Rechtsvisualisierung im Strafrecht; Boehme-Neßler, Unscharfes Recht.

5 Vgl. dazu auch Holzer, Rechtsvisualisierung im Strafrecht, S. 5 f.

6 Deutsches Sprichwörter-Lexikon, 1987, S. 378.

7 So Thüsing, in: NVwZ 2013, S. 193 (193) mit weiteren guten Ansätzen in seinem Aufsatz, der in der Vergangenheit aus einem anderen Blickwinkel bereits exemplarisch von Großfeld u.a., für die Bildende Kunst letztmalig von Bredekamp, in: Hohe Luft, Ausgabe 2/2012, S. 68 (71) und grundlegend auch von Sachs-Hombach, Das Bild als kommunikatives Medium, behandelt wurden.

8 Kaum ein Studierender hat das Lehrbuch von Larenz-Canaris, Schuldrecht II/2, in der letzten erschienenen 13. Auflage, 1994, gelesen - eine Neuauflage lässt bis heute auf sich warten; Esser-Weyers Lehrbuch ist trotz seiner Qualitäten letztmalig in der 8. Auflage, 2000, aufgelegt worden und nach der Schuldrechtsreform nicht mehr erschienen. 
stetig steigende Informationsflut ${ }^{9}$ und zum anderen durch die Flut an Visualisierungen in der Umwelt und in ihrer Entwicklung an eine andere Art der Wissensaufnahme gewöhnt bzw. herangeführt worden. Diese Art der Wissensaufnahme komplett mit Beginn eines Rechtsstudiums (egal ob grundständige Universität oder Universität der angewandten Wissenschaften) auszuschalten, ist nahezu unmöglich, ja in der Summe sogar unproduktiv. Unproduktiv ist dies deshalb, weil man mit dem nicht ausgeschöpften Nutzen und dem möglichen Mehrwert der neuen technologischen Mittel, die Zeichen der Zeit missdeutet, indem man sich die aktuellen und zukünftigen von der Rechtsvisualisierung angebotenen Hilfsmittel, auch fachdisziplinübergreifend, nicht zu eigen macht und diese aus dem Lehr- und Lernprozess ausklammert. Diese notwendige Reflexion wollen bisher auch die mit vorherrschender Visualisierung in der Umwelt aufgewachsenen Lehrstuhlinhaberinnen und -inhaber, trotz ihres Wissens um diese Faktoren, bisher noch nicht beschreiten. Ursache hierfür ist möglicherweise der immer noch belastende und traditionell anerkannte Satz, dass nur die Linearität, also ein DINA 4 hochkant i.d.R. mittels einer Textverarbeitung vollgeschriebenes Papier ein belegbarer Faktor für (Rechts-)Wissenschaft sein kann. ${ }^{10}$ Farben und Visualisierungen sind hier nach gängiger wissenschaftlicher Ansicht wohl Fehl am Platze. „Die Rechtswissenschaft(en) hinken leider sehr oft innovativen Methoden hinterher, „da sie oft statisch sind und alles Nichtlineare als nicht wissenschaftlich ablehnen ". ${ }^{11}$ Aus der Gehirnforschung ist jedoch allgemein bekannt, dass unsere menschliche Wahrnehmung grundsätzlich nichts mit Objektivität zu tun hat. ${ }^{12}$ Gerade der soziale Konstruktionismus ${ }^{13}$ zeigt uns auf, dass jeder sich seine eigene individuelle Realität erschafft. ${ }^{14}$ Das mit Buchstaben vollgeschriebene gleiche Blatt Papier bedeutet mit seinen Zeichen für den Leser A eine andere soziale Wirklichkeit als für den Leser B. Die Neutralität des Papiers und der Buchstabenzentrierung erscheint somit nur vordergründig. Es sind Visualisierungen (vereinfacht ausgedrückt die Bilder), die jedes Individuum durch das Lesen hervorbringt. Beides, sowohl der soziale Konstruktionismus als auch die Gehirn- und Kommunikationsforschung sprechen von Bildern oder visuellen Produkten, die Menschen durch das Lesen von linearen Texten produzieren und subjektiv ausdeuten. Um dieses Geflecht, wie wir zum einen Rechtskommunikation und zum anderen Lehr- und Lernprozesse definieren, aufzubrechen, sollte der Umweg von der Linearität zur Visualisierung (wie auch immer wir diese ,juristischen Bilder“ in unserem Kopf bezeichnen wollen)

9 Exemplarisch kann hier, neben der allgemeinen Wissensflut, auch die Ausweitung durch zahlreiche Entscheidungen der verschiedenen, nicht nur der Arbeitsgerichtsbarkeit, von landesweiten zu europaweiten und internationalen Entscheidungen Erwähnung finden, was aus meiner Sicht ein stetiger Wandel zum Case Law bedeutet.

10 Möglicherweise auch dieser Artikel? sic!

11 Khalil, Rechtsvisualisierung durch Mindmaps, S. 473.

12 Vgl. m.w.N. auch zu den Anfängen der Gehirnforschung ebenda.

13 Gergen, Konstruierte Wirklichkeiten, passim; Gergen/Gergen, Der soziale Konstruktionismus, passim.

14 Im Anschluss an Gergen für alle Winter, Sozialer Konstruktionismus, S. 124: „Der Konstruktionismus betrachtet die Sprache als eine Form sozialen Handelns. Sie drückt nicht Emotionen oder Denken auf sekundäre und passive Weise aus, sondern gestaltet und artikuliert sie. Sprache bildet nicht die Wirklichkeit ab, sondern bringt diese hervor.". 
beschritten werden. Dieser grundlegende Paradigmenwechsel, weg von einer vermeintlichen Bilderscheu und hin zu einem akzeptiertem Umgang mit zukunftsträchtigen Visualisierungen, sollte in den nächsten Jahren angestrebt werden, denn für die nachkommenden Generationen müssen geeignete Hilfsmittel geschaffen werden, die es ihnen ermöglichen, das juristische Studium und den späteren Beruf in der gegenwärtigen medial und visuell expandierenden Welt zu bewältigen. Diese Anpassungen an die Erfordernisse der Gegenwart und für die Zukunft sollten auch in der juristischen Lehre Beachtung finden. Den Lehrenden muss es ohne größere Kritik im Innenverhältnis ermöglicht werden, sich den Zeichen der Zeit anzupassen und rechtsvisualisierende Elemente in den Lehralltag implementieren zu dürfen, ohne dass es möglicherweise „wissenschaftliches Ungemach“ zur Folge hat. ${ }^{15}$

\section{Wieso Visualisierung in der Jurisprudenz?}

Mit Hilfe der Visualisierung ist eine wissensökonomische Erweiterung in der Rechtswissenschaft denkbar, um neuen, mediensozialen, personenbezogenen Grenzsituationen gerecht zu werden, die möglicherweise durch die Linearität der Schriftsprache als Kommunikationsmittel so augenscheinlich nicht oder für die lineare Kommunikation in der Zukunft allein nicht mehr zu bewältigen sind. ${ }^{16}$

„Das Bild verdeutliche, was immer auch unbestimmt bleibe, Idealität und Bedeutung. Zeit und Handlung können sie nur nichtsprachlich durch Bildebene, Bildraum, Farbe und Form, ausdrücken. [Und] Bildlichkeit eignet längst nicht nur dem Visuellen allein". 17

Da die Natur oft visuell arbeitet und ihre Ideen auf der Visualisierung aufbaut, findet auch Leibniz, dass die Ideen, die der Natur nachempfunden sind, die zuverlässigsten und nützlichsten sind. ${ }^{18}$

Dies würde für die Linearität des Wissens bedeuten, dass das Visuelle nicht länger als Möglichkeit der Wissensvermittlung zu ignorieren ist und alle Worte durch Visualisierungen, z.B. multisensorische Darstellungen zu ersetzen sind, die nur noch gebündelte Rechtsbegriffe oder aber zukünftig keine geschriebenen Wörter mehr enthalten. Hierbei müssen neue Wege der Visualisierung mit einbezogen werden, die über eine Ikonisierung hinauszugehen haben. ${ }^{19}$

Leibniz hat bereits detailliert die Fehlerhaftigkeit der Worte dargestellt. Der Transfereffekt der Fehlerhaftigkeit zur juristischen Sprache und zur lex scripta ist bereits

15 Dazu zählt die Implementierung von visuellen Elementen in Klausuren, zumeist als Bildverstärker. Ansätze zur Implementierung in Klausuren gab es bereits 2003 in Tutorien an der Johann Wolfgang Goethe-Universität, aber auch sehr ausführlich durch Röhl/Ulbrich, Recht anschaulich, S. 14, in einem von der Volkswagen-Stiftung und der Ruhr-Universität Bochum geförderten Projekt.

16 Khalil, Visuelle Jurisprudenz, S. 32.

17 Westerkamp, in: Zeitschrift für Ästhetik und Allgemeine Kunstwissenschaft, 58 (2013), S. 35 ff.; vgl. auch die Besprechung von Gross, in: FAZ vom 15.5.2013, S. N3, ohne dessen Besprechung der Aufsatz nicht einem größeren Publikum zugänglich gemacht worden wäre.

$18 \mathrm{Zu}$ Leibniz' Fehlerhaftigkeit der Worte vgl. ausführlich Khalil, Visuelle Jurisprudenz, S. 30 ff.

19 Vgl. auch Khalil, Neue Zeichen und neue Bilder, passim. 
aufgezeigt worden. ${ }^{20}$ Mithin bedarf es lediglich einer Rückbesinnung auf die Tatsache, dass die Worte fehlerhaft sind. Ein Wandel im Denken der Rechtswissenschaften ist nunmehr eingetreten. Statt einer tiefen tradierten Ablehnung der neuen visualisierenden Möglichkeiten sollte ein grundständiger Lehrstuhl für Rechtsvisualisierung und Rechtskommunikation geschaffen werden und sogenannte Rechtskommunikatoren sollten im fachdisziplinären Austausch angepasste Lehr- und Lernmethoden erarbeiten und implementieren. ${ }^{21}$ Neben den klassischen Juristinnen und Juristen müssen Rechtsinformatikerinnen und Rechtsinformatiker, Designerinnen und Designer und Ingenieurinnen und Ingenieure gemeinsam die weiteren Grundlagen für den lehr- und lerntechnischen Fortschritt in der Rechtswissenschaft entwickeln. ${ }^{22}$ Es ist zu konstatieren, dass die erfolgreiche Implementierung der Visualisierung in den Rechtswissenschaften derzeit wohl nur „Hand in Hand“ mit einer technikaffinen Zukunft realisierbar ist. ${ }^{23}$ Das ist kein Rückschritt in die Zeiten des pictorialen Analphabetismus des Mittelalters, wie des Öfteren als Gegenargument angeführt wird, sondern ein Schritt zur Synthese von Schrift und visualisierten Elementen für den Lehr- und Lernprozess. ${ }^{24}$

\section{Was ist Visuelle Jurisprudenz? - Eine kurze Begriffsdefinition}

In der derzeitigen Diskussion werden die Begriffe Rechtsvisualisierung, visuelle Jurisprudenz und multisensorisches Recht teils als Synonyme, teils als Untergruppe des jeweils anderen Begriffs, teils als erweiterte Modifikation der anderen Begriffe durch Implementierung weiterer zum Einsatz kommender Sinne und Systeme verstanden.

\section{a. Visuelle Jurisprudenz}

Am Anfang war es die Rechtsvisualisierung, die versucht hat, in dem linearen Alltag der Rechtswissenschaft(en) visualisierte Darstellungen anzubieten, die es den Lernenden, aber auch den Lehrenden ermöglichen, die Stofffülle so zu komprimieren und verständlich zu machen, dass einer Überlastung des menschlichen Verstandes und seiner Ressourcen entgegengewirkt werden kann.

Visuelle Jurisprudenz ist also das aus Sicht des Autors anzustrebende Ziel einer völligen Visualisierung mittels immer wieder neu implementierter technologischer Möglichkeiten zur Ablösung der schriftsprachlichen Kommunikationsprobleme. Diesem Ansatz „liegt die Annahme zugrunde, dass die heutigen Rechtsnormen nicht nur linear darstellbar sind, sondern auch visualisierbar sind und zwar mittels Rezeption

20 Vgl. Khalil, Visuelle Jurisprudenz, passim.

21 Dieser Ansatz ist bereits auf der Münchener Tagung „Recht \& Logik“ 2012 vor dem internationalen Fachpublikum angesprochen worden, vgl. dazu auch Khalil, Neue Zeichen und neue Bilder, S. $968 \mathrm{f}$.

22 Zum Überblick der verschieden eingesetzten Termini in diesem Bereich vgl. Holzer, Rechtsvisualisierung im Strafrecht, S. 58.

23 Kritisch dazu wohl Bengez: „Das Recht war und ist ein normatives Praxissystem, das in den letzten Jahrhunderten textuell überformt wurde um sich nunmehr in ein maschinendominiertes Herrschaftssystem zumindest partiell zu wandeln." vgl. http:// rechtundlogik2012.quantius.org/ (9.1.2014).

24 Vgl. auch Khalil, in: Schweighofer/Geist et al. (Hrsg.), S. 468 (473). 
von Bildern mit juristischem Inhalt sowie durch die Nutzung moderner Visualisierungsmöglichkeiten “.25

\section{b. Rechtsvisualisierung}

Rechtsvisualisierung ist „die optische Gestaltung rechtlicher Inhalte durch Generierung oder Instrumentalisierung von Bildern zur rechtsinternen Kommunikation “. ${ }^{26}$ Besser ist es, diese Definition neben den „Bildern“ auch auf modernste Techniken zu erweitern.

Rechtsvisualisierungen werden vermehrt in der Lehre eingesetzt, z.B. in Form von Visualisierungen im Privatrecht, im Prozessrecht und sehr gerne auch im Strafrecht. Hier sei jedoch ausdrücklich angemerkt, dass zwischen professionell aufgearbeiteten Visualisierungen und eher profanen Comics unterschieden werden sollte, die keine visualisierende Verstärkung der juristischen Aussage beinhalten, sondern vielmehr als Effekthascherei schmückendes Beiwerk darstellen. ${ }^{27}$

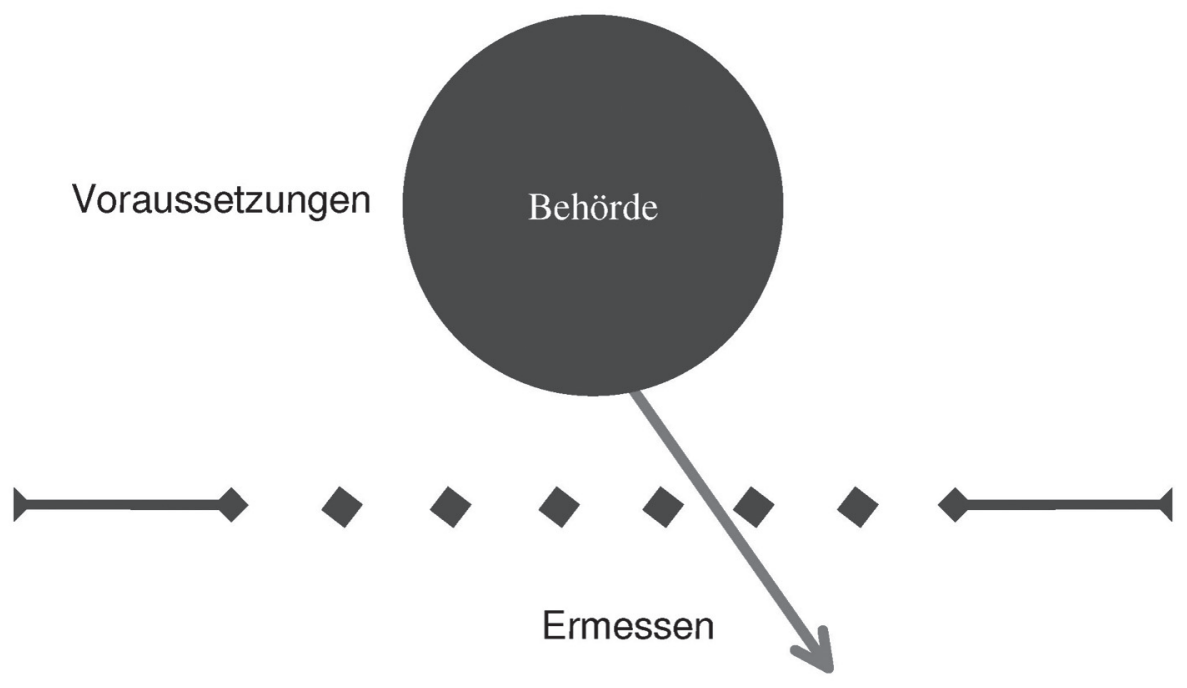

(c) Friedrich Lachmayer

Abb. 2: Ermessen von Friedrich Lachmayer, www.legalvisualization.com (12.3.2014)

25 So Khalil, Visuelle Jurisprudenz, S. 30.

26 Eine korrespondierende Definition findet sich im Anschluss an Hilgendorf, Beiträge zur Rechtsvisualisierung, bei Holzer, Rechtsvisualisierung im Strafrecht, S. 58.

27 Wissensökonomisch sind die Visualisierungen von Heindl/Kablig et al., WGG II strukturiert, passim, oder von Lachmayer (vgl. Abb. 2), Nachweise bei www.legalvisualization.com (12.3.2013); aber auch Pridik, vgl. www.npridik.de (12.3.2013); meine anfänglich erst positiven Äußerungen zu Comics haben sich durch die wesentlich effektiveren und aussagefähigeren Möglichkeiten der Visualisierungen im Recht, weg von den Comics und hin zum Legal Design, gewandelt, vgl. zum Legal Design für die Praxis auch exemplarisch Heindl/Kablig et al., WGG II strukturiert. 


\section{c. Multisensorisches Recht}

Multisensorisches Recht ${ }^{28}$ ist die Einbeziehung einer Vielzahl von Sinnen, die dem Menschen zur Verfügung stehen, um das lineare Wissen zu ergänzen und dieses je nach Vorliebe des einzelnen Menschen für den Lehr- und/oder Lernprozess einzusetzen.

Der Begriff des multisensorischen Rechts soll über das reine Sehen hinaus auch alle anderen Sinneswahrnehmungen in ihrer rechtlichen Relevanz erfassen und geht damit über den der Rechtsvisualisierung hinaus. Das multisensorische Recht ist somit initiiert worden, weil der Begriff der Rechtsvisualisierung nicht alle einschlägigen Dimensionen der Visualisierung abbilden kann. ${ }^{29}$ Es ist sowohl Großfeld, ${ }^{30}$ Eichenhofer, ${ }^{31}$ als auch Brunschwig ${ }^{32}$ zu verdanken, die erstmalig Sinne in Form von gustatorischen, olfaktorischen und kinästhetischen Systemen, neben den klassischen Sinnen und somit zum etablierten Begriff der Rechtsvisualisierung, unbewusst oder auch nicht, ergänzten.

Der vorliegende Aufsatz wendet sich zunächst ausschließlich der „grundlegenden“ Rechtsvisualisierung zu, um die bisherigen Möglichkeiten und Ergebnisse einer Visualisierung darzustellen. Im Folgenden soll dann auch der multisensorische Aspekt in die Erwägungen einbezogen werden.

\section{Status Quo der Rechtsvisualisierung}

Man muss nicht lange überlegen, um rechtsvisualisierende Elemente im Arbeitsalltag eines Dozenten zu erkennen: Denkt man zurück an das erste Studiensemester im Fach „Bürgerliches Recht“, als der Rechtsprofessor/die Rechtsprofessorin versuchte, den Studierenden das Abstraktionsprinzip bzw. Vertragsverhältnisse im Dreipersonenverhältnis näher zu bringen. Die überwiegende Anzahl von Studierenden verstand im klassischen Vorlesungsvortrag nicht viel. ${ }^{33}$ Erst als der Dozent ein sogenanntes „Beziehungsdreieck“ an die Tafel malte, hatte man das Gefühl, es verstanden zu haben.

28 Ausführlich dazu und mit weiteren Erläuterungen zur Entstehungsgeschichte bei Lachmayer/Hofmann et al., Multisensorische Modelle des Rechts.

29 Es wird allgemein die Ansicht vertreten, dass sich „der Begriff "Rechtsvisualisierung" als zu eng erweist, weil er weder dem audiovisuellen noch dem multisensorischen Recht gerecht wird, vgl. dazu nochmals Lachmayer/Hofmann et al., Multisensorische Modelle des Rechts.

30 Großfeld, in: NJW 1994, S. 191 ff. und auch ders., in: ZVglRWiss 2010, S. 379 (383): Großfeld zitiert das australische Mabo-Urteil (Mabo and others v. The State of Queensland [Nr. 2, (1992) 175 Commonwealth L. Rep. 1; vgl. auch die identische FN 98 bei Großfeld), das „eigenständige Landrechte der Ureinwohner anerkennt und bezieht sich dafür auf die Verbindung zwischen Gesängen und Land. Die Gesänge sind heute anerkannt als Rechtsquelle.“.

31 Eichenhofer, in: NJW 2008, S. $2828 \mathrm{ff}$.

32 Brunschwig, in: FS Schweighöfer, S. 573 ff., die den Begriff Multisensory Law in die internationale Fachwelt eingeführt hat.

33 Viele Dozenten behaupten auch heute noch, dass zahlreiche Bachelorabsolventen auch nach ihrem Studium grundlegende Prinzipien nicht verstanden bzw. verinnerlicht haben. 


\section{Grafische Darstellung}

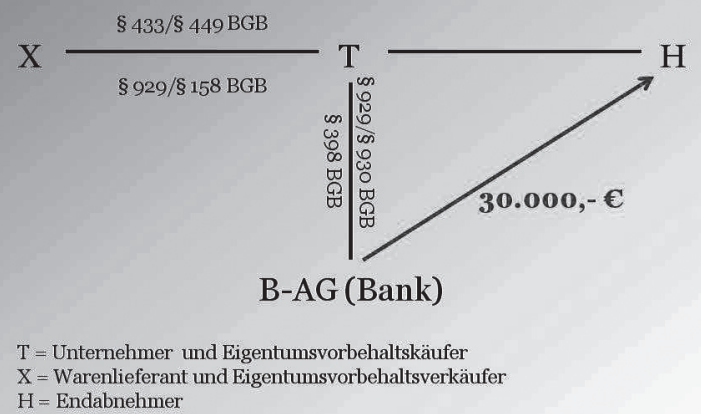

Abb. 3: Beziehungsdreieick zur Globalzession von Aiman Khalil

Wieso gestaltet sich die Einprägsamkeit von linearem Wissen für viele Studierende so schwer? Warum ist die Wissensvermittlung durch die Zuhilfenahme von visualisierten Elementen - von der einfachen Grafik zum Film, bis zur mehrdimensionalen Technik für die Generationen der Studierenden nachhaltiger, als die traditionelle Vermittlung des linearen Lesestoffs?

Stimmen in der Literatur ${ }^{34}$ führen aus, dass nun Bildungsschichten die Universitäten und Hochschulen bevölkern, bei denen der von zu Hause mitgebrachte „Bildungsrucksack " nicht dem vergangener Generationen von ausschließlich Studierenden aus dem Bildungsbürgertum entspricht. Dieser Grund kann nach aktuellen Erhebungen nicht als einziger für den Wandel verantwortlich gemacht werden. Die überwiegende Anzahl der Studierenden ist nach neuesten Statistiken ${ }^{35}$ weiterhin der Oberschicht und dem Bildungsbürgertum zuzurechnen. Die geringe Anzahl der Studierenden, die nicht den beiden genannten Schichten angehören, ist immer noch prozentual sehr niedrig. Es sind vornehmlich die Kinder der Oberschicht und des Bildungsbürgertums, deren fehlender „Wissenspool“, trotz des vermeintlichen Bildungsvorteils, sowohl in sprachlicher als auch in denklogischer Hinsicht nur noch eingeschränkt die linearen Voraussetzungen der heutigen Zeit für ein grundständiges Studium der Rechte mitbringen. Allerdings ist der wissensspezifische Hintergrund der heutigen Studentinnen und Studenten ein deutlich anderer und die Vorgehensweise, wie Wis-

34 Fehlgehend m.E. die Ansicht von Röhl/Ulbrich, Recht anschaulich, S. 24 ff., die dies aufgrund neuer Bildungsschichten an Universitäten und Hochschulen begründen, was durch neue Studien und Erfahrungswerte (Stichwort: fehlende Kompetenzen auch von Kindern des Bildungsbürgertums) in der Lehre widerlegt ist. Richtig dagegen ist deren Ansatz, dass „wer zusätzlich zur Schreibkompetenz über Bilderkompetenz verfügt, auf Dauer im Vorteil [ist]“, S. 25.

35 Schindler, Aufstiegsangst?. 
sen erworben und gesammelt wird, ist offenkundig multimedialer und globaler als in den vergangenen Generationen. ${ }^{36}$

Dieser Status quo ist m.E. auf den Wandel in der Wissensaufnahme der Studierenden zurückzuführen. Hier ist auch ein „Zurückrudern“ zu alten Standards nicht mehr sinnvoll. Vielmehr sollten neue lehr- und lerndidaktische Wege zu den bestehenden in einer Synthese hinzugefügt werden, die dem Dilemma der Notwendigkeit einer zeitgemäßen Lehre und dem stetig steigenden und technikversierten Verständnis der Studierenden entgegen kommt.

Was für Möglichkeiten für eine visuelle Unterstützung der Lehre in Frage kommen, soll im Folgenden exemplarisch an ausgewählten Beispielen deskriptiv dargestellt werden. 37

\section{a. Beispiel 1: Der Einsatz von computerunterstützen und handschriftlichen Mind Maps}

Zwischen den Jahren 1998 und 2010 etablierte sich für die damalige Zeit eine interessante und effektive Lehr- und Lernmethode, die sich am Anfang nur zögerlich in den Lehr- und Lernbereich der Juristen einfügte. Neben dem Clustering, das zunächst in der Pädagogik und in den Wirtschaftswissenschaften als Neuerung hochgelobt wurde, entwickelte sich parallel dazu das Mindmapping, welches nun auch in den Rechtswissenschaften erfolgreich Einzug hielt.

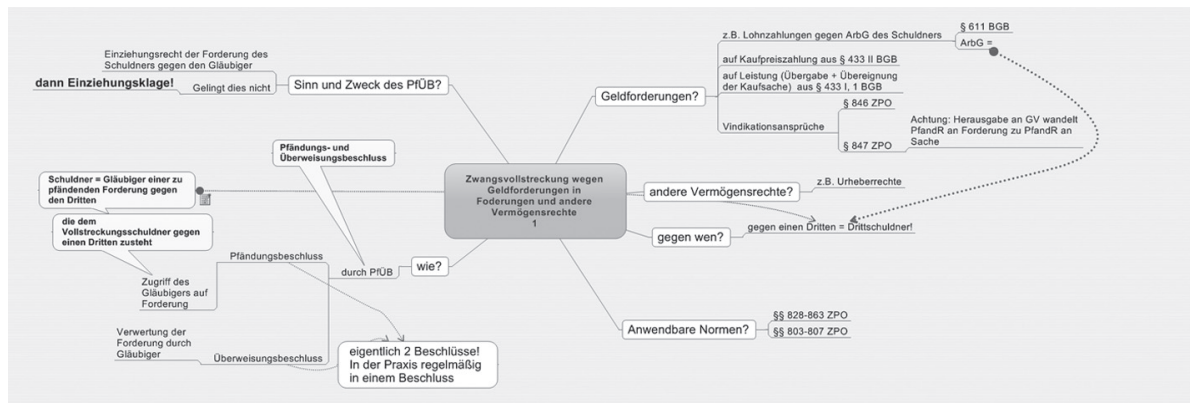

Abb. 4: Khalil, Zwangsvollstreckung wegen Geldforderungen und andere Vermögensrechte

Doch wo steht das Mindmapping in der Rechtslehre heute? Wird es immer noch (mit Enthusiasmus) angewandt oder war es nur ein sogenannter „Hype“, der nun abklingt bzw. vorbei ist? Anfang der 90er Jahre begann der Siegeszug der Rechnerwelt in den

36 Hinzu kommt die kontinuierlich zugenommene Stoffmenge, mit der sowohl die Lehrenden als auch die Lernenden konfrontiert sind; vgl. exemplarisch den Umfang des aktuellen Medicus/Petersen, Bürgerliches Recht, 24. Auflage, München 2013, zum damaligen Medicus, Bürgerliches Recht, 8. Auflage, München 1978.

37 Es sei angemerkt, dass es mittlerweile eine Fülle an interessanten Lösungsansätzen gibt, aber auch verbesserte Altlösungen sowie in der Entwicklung befindliche Ansätze. Ich habe mich entschlossen, nur wenige der m.E. derzeit machbaren Lösungen für diesen Beitrag in aller Kürze vorzustellen. 
juristischen Seminaren. ${ }^{38}$ Die Etablierung von einfachen 286er- und 386er-Prozessoren mit 40 Mhz-Taktung in PC's (teilweise schon mit 10 MB Festplattenkapazität) schritt voran und bildete auch für computerunterstützte Mindmaps die technische Grundlage. Zu dieser Zeit nutzten viele Studierende und Lehrende noch die klassische Schreibmaschine, um ihre Hausarbeiten und Seminararbeiten zu bewältigen, da der Kostenfaktor eine nicht unerhebliche Rolle spielte. Wie mir berichtet wurde, hat ein erheblicher Teil der Studierenden noch ihre Hausarbeiten in handschriftlicher Form zur Benotung abgegeben. Besondere Lernmethoden wie das Clustering im Studentenalltag oder im Dozentenalltag von Juristen einzusetzen, war gänzlich unbekannt.

Zur damaligen Zeit war der Begriff des Mindmappings noch nicht kreiert. ${ }^{39}$ Jedoch das Clustering hatte sich bereits etabliert und vornehmlich in der Pädagogik bei den Lehrerinnen und Lehrern an allgemeinbildenden Schulen Eingang gefunden. ${ }^{40}$

Das Mindmapping ${ }^{41}$ ist inzwischen im Alltag der Studierenden und Lehrenden angekommen. Es hat sich durchgesetzt und wird ohne großes Aufsehen eingesetzt, wobei sich zwei Fraktionen gebildet haben. Die eine Personengruppe, die mit der Methode nichts anfangen kann und andere Lernmethoden einsetzt und die andere Gruppe, die diese Lehr- und Lernmethode mit Enthusiasmus für das jeweilige Ziel einsetzt bzw. einsetzen möchte. Hierbei ist zu beachten, dass juristische Mindmaps oft komplexer zu gestalten sind, als die klassischen Mindmaps. ${ }^{42}$ Juristische Mindmaps gestatten mehrtextuale Äste, Zweige und Verzweigungen sowie Anmerkungen.

38 Exemplarisch für viele: Weyers, der mit viel Einsatz an seinem Fachbereich in Frankfurt am Main die Vernetzung und Einführung von juristischen Datenbanken mit der EDV vorantrieb.

39 Buzan/Buzan, Das Mind-Map Buch, passim; Buzan rühmt sich, diese Lernmethode und den Begriff als erster in seinem Werk Buzan/Buzan, Mind-Map, implementiert zu haben, was für das Clustering und die eigentliche Methode sicherlich nicht der Fall war, da diese Methode schon in den 70er Jahren in der Pädagogik nicht unbekannt war. Zumindest ist ihm und seinem Band zugute zu halten, maßgebliche Impulse zum Bekanntheitsgrad dieser Methode zunächst im angloamerikanischen Bereich und später weltweit gefördert zu haben.

40 Vgl. Khalil, Rechtsvisualisierung durch Mindmaps (k)eine Modeerscheinung?, S. 468 ff. m.w.N.

41 Die Schreibweise ist teilweise uneinheitlich. Der Begriff JuraMindMaps ist urheberrechtlich geschützt, vgl. www.cfmueller-campus.de; der Begriff Mind-Map ist ebenfalls urheberrechtlich von Tony Buzan geschützt. Ich verwende hier die offenen Begriffe Mindmap und Mindmapping.

42 Die klassische Mindmap sollte grundsätzlich immer nur mit einem Wort oder Begriff pro Ast und Zweig versehen werden. 


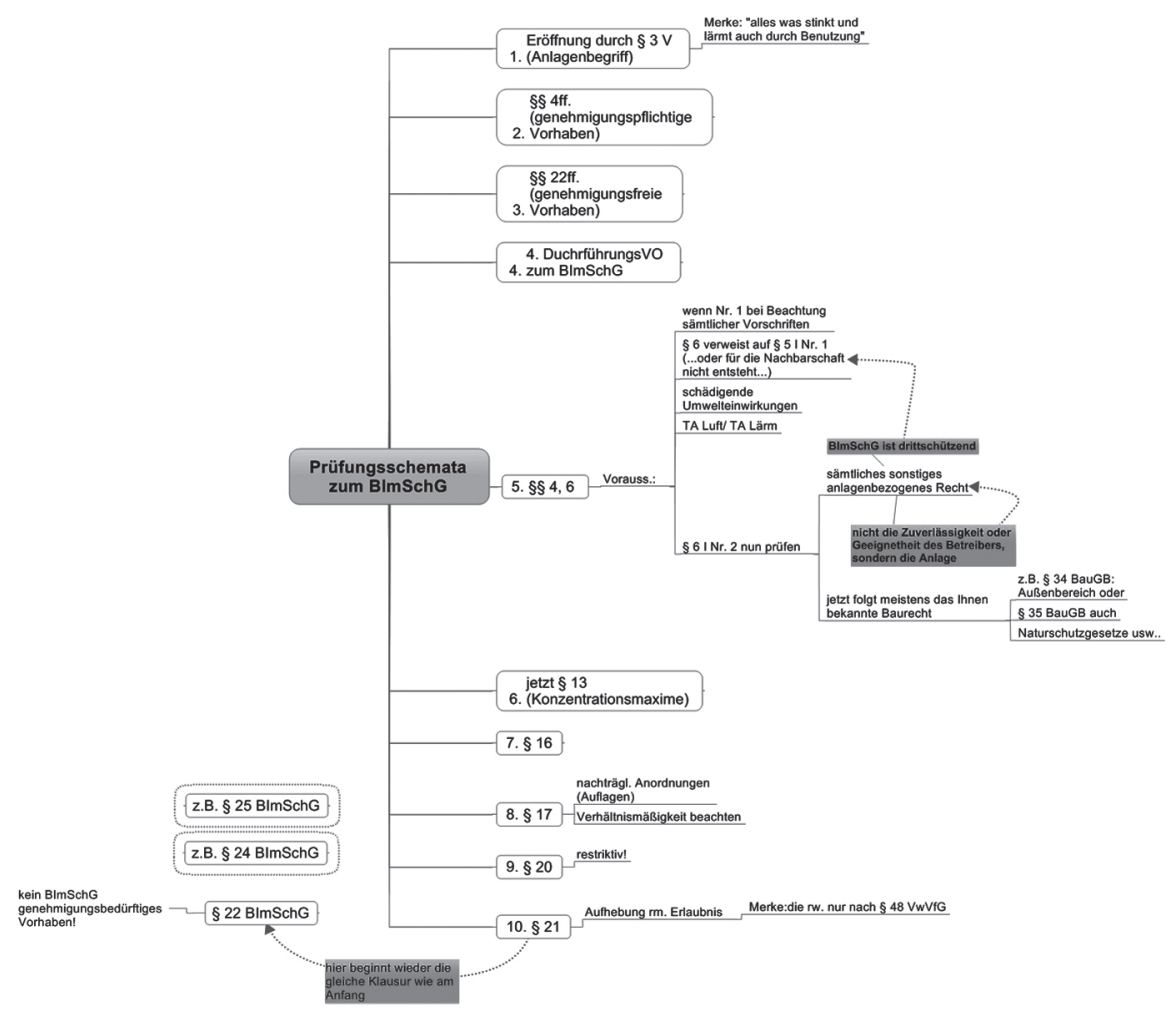

Abb. 5: BImSchG von Aiman Khalil

Wünschenswert wäre es weiterhin, wenn die Universitäten und Hochschulen neben ihren Repetitorien auch von fachlich ausgebildetem Personal Kurse zu dieser Methode für Interessierte anbieten würden, wobei auf den Studiengang bezogene, spezialisierte juristische Mindmaps gelehrt werden sollten. 


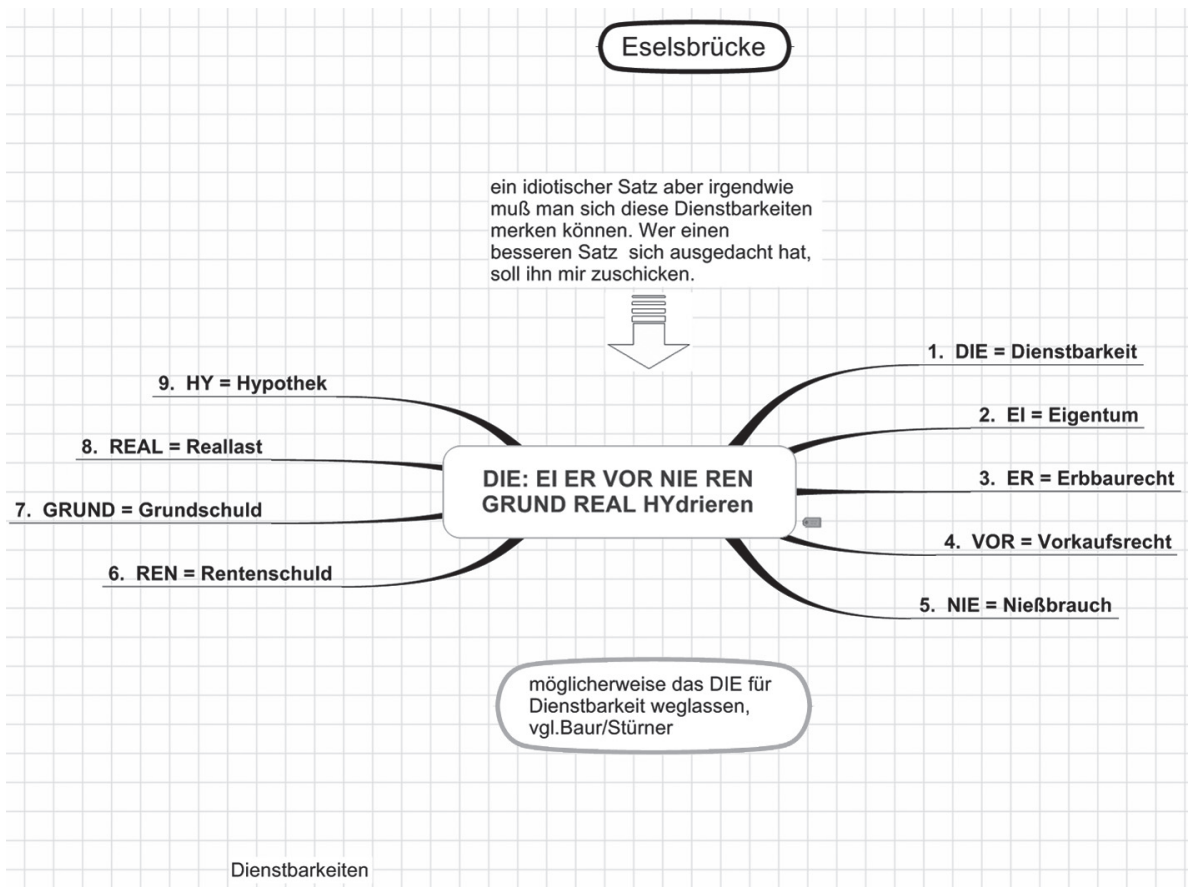

Abb. 6: Eselsbrücke Dienstbarkeiten von Aiman Khalil

Diese Kurse müssten organisiert und zudem unterstützt werden bei der Weiterentwicklung solcher Methoden durch einen bereits postulierten neuen Lehrstuhl, resp. durch eine Professur an den Hochschulen für angewandte Wissenschaften.

\section{b. Beispiel 2: Der Skripteneinsatz mit visualisierten Elementen}

Skripten gehören mittlerweile zum Alltag von Studierenden der Rechte an Universitäten, als auch an Fachhochschulen. Gleich vorab, eine Ablehnung von Skripten wird hier nicht vertreten. Vielmehr sollten Skripten dann eingesetzt werden, wenn diese nach dem Lesen eines ordentlichen Lehrbuchs, ${ }^{43}$ verbunden mit dem glücklichen Umstand, eine gute Vorlesung zum gleichen Themenkomplex besucht zu haben, einen wiederholenden und in das Gedächtnis einprägenden Charakter besitzen. Erst dann ist ein effektives Wiederholen mittels Skripten (ob nun effektiver mit externem Repetitor oder ohne, sei dahingestellt) grundsätzlich garantiert. ${ }^{44}$

43 Trotz des mittlerweile großen Umfangs mancher Skripten, gibt es m.E. qualitative Unterschiede zu den vom Umfang regelmäßig umfassenderen Standardlehrbüchern der etablierten Rechtsverlage. Viele der Skripten geben eine zitierte Kurzbeschreibung von aktuellen und ehemaligen Lehrbüchern wieder und eignen sich deshalb m.E. für den schnellen Überblick sehr gut.

44 Mittlerweile verfügen die meisten juristischen Fakultäten über eigene und effektive Repetitorkurse, z.B. in Form von Studienprofessuren wie exemplarisch an der Uni Passau, was ein erster richtiger Schritt ist. 
Die Inhalte dieser Skripte werden noch wesentlich einprägsamer, wenn noch vermehrt visuelle Abbildungen implementiert werden. Der Wunsch, Visualisierungen verstärkt auch in Standardliteratur einzubinden, scheitert derzeit nicht an den Autoren, aber leider immer noch am Unwillen der führenden Rechtsverlage und der damit verbundenen Kostenfrage. ${ }^{45}$ Insbesondere spezielle Infografiken und von Designerinnen und Designern und Juristinnen und Juristen gemeinsam entwickelte „Legal Designs“ sind immer noch Mangelware in gebundener Literatur, wenn man die üblichen Tabellen und die teilweise im Erbrecht vorfindenden Darstellungen für die Erbenfolge vernachlässigt. ${ }^{46}$ Aber auch der Einsatz von Farben und deren Wirkung insbesondere auf juristische Texte wird immer noch zu sehr unterschätzt. Hier sind Farbsysteme, die Systematik der Farbanwendung und die Farben an sich von hoher Bedeutung. Es hat sich eine Farbenstruktur in Anlehnung an die jeweilige Rechtsdisziplin entwickelt. Aus der systematischen Farbverwendung im Recht ergeben sich folgerichtig auch Konsequenzen für die Gestaltung im Bereich der Rechtsvisualisierung. ${ }^{47}$ Es sei angemerkt, dass Farben auch als Mittel der visuellen Informationskodierung dienen können. ${ }^{48}$ Wichtig zu wissen ist, dass wir hier nicht von Visualisierungen sprechen, die lediglich einem ästhetischen Hintergrund besitzen und eine nur optische Bereicherung des Textes beinhalten. Vielmehr geht es um Visualisierungen im engeren Sinne. Dies sind auf Grund der derzeit noch beschränkten Möglichkeiten Visualisierungen, die dem Text dienen und eine Synthese bilden. Diese dienende Funktion wird immer mehr durch eigenständige, selbsterklärende und textunabhängige Visualisierungen abgelöst werden.

45 Der derzeit machbare Weg des digitalisierten Lehrbuchs, mit zahlreichen Visualisierungen, erfolgt durch die Verbreitung mittels einer App und dem Lesen auf Tablets und anderen Rechnern.

46 Staudingers Eckpfeiler des Zivilrechts enthält in der mir vorliegenden Bearbeitung 2005 nicht ein einziges Schaubild, geschweige denn eine Tabelle; selbst der erbrechtliche Teil enthält keine unterstützende Visualisierung.

47 Dazu eingehend bereits Mielke/Wolff, Welche Farbe hat das Recht?, passim.

48 Vgl. dazu die Farbsysteme von Silvestrini/Fischer, Farbsysteme in Kunst und Wissenschaft, passim. 


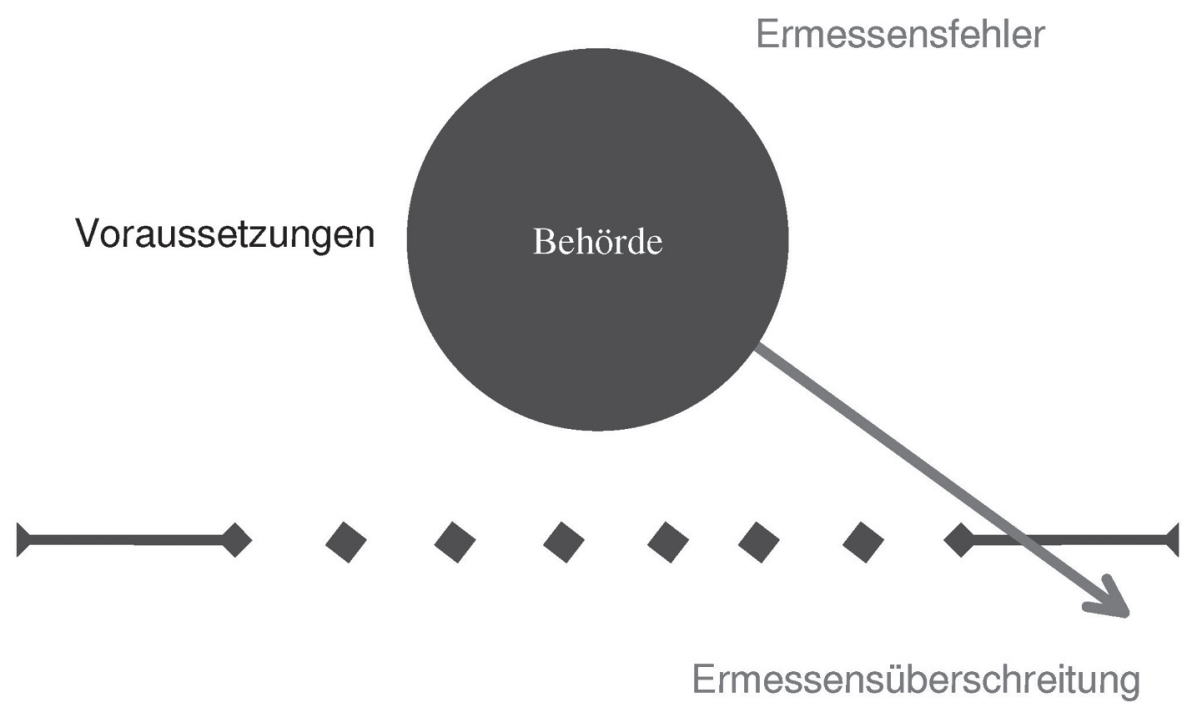

(c) Friedrich Lachmayer

Abb. 7: Ermessensüberschreitung von Friedrich Lachmayer (vgl. www.legalvisualization.com, 19.2.2014)

In der Quintessenz für die heutige Zeit werden sich die Verlage mittel- und langfristig selbst den bisherigen Markt für anspruchsvolle juristische Standardliteratur beschränken, wenn sie sich den visuellen Elementen, dazu gehört auch der Einsatz von farbigen Grafiken, Abbildungen etc., weiterhin verschließen. Denn, nur farblose und visualisierungsscheue Produkte werden nicht mehr durch die neuen Generationen an Fachlesern gekauft und gelesen. Hier sollte ein Umdenken schnellstmöglich erfolgen. ${ }^{49}$ Hier sei angemerkt, dass durch den Wandel, hin zum elektronischen Vertrieb, auch verstärkt Visualisierungen in die Werke implementiert werden können. ${ }^{50}$

49 Der C.H. Beck Verlag hat für seine renommierte Zeitschrift NJW einst einen eher farblosen Versuch gestartet und wohl aus Kostengründen wieder aufgegeben. Zumindest wird bei den Zeitschriften versucht, mittels „Zweifarbigkeit“ (einer weiteren Druckfarbe, zumeist ist es neben der schwarzen Schrift nun auch rot) einen eher dürftigen ersten und unbewussten Schritt weg von der Linearität zu wagen.

50 Auf dem Weg, den Wandel zu vollziehen, ist der Haufe Verlag, der heute 95\% seines Umsatzes mit digitalen und visualisierungsreichen Produkten macht, vgl. dazu Giersberg, in: FAZ vom 28.2.2014, S. 21. Auch der Beck-Verlag besitzt seit Jahren online-Datenbanken, die bequem mit Visualisierungen gefüttert werden könnten. 


\section{c. Beispiel 3: Der Einsatz von Präsentationshilfsmitteln in der Lehre}

Die Applikationen „PowerPoint“ und „Prezi“51 haben den Vorlesungsalltag schon seit Jahren erreicht. Kaum ein Dozent liest mehr klassisch in der Vorlesung, sondern setzt zur Visualisierung verstärkt Folien ein. Leider sind diese Folien mit linearem Text überlastet und nur wenige Grafiken finden Zugang in den Vorlesungsalltag.

Viele Dozenten beklagen, dass sie die große Fülle an Lernstoff nicht mit einer visuellen Darstellung und einer grafischen Reduzierung auf das Nötigste darstellen können. Auch hier bedarf es der Unterstützungshandlung von Rechtskommunikatoren, die einprägsame Visualisierungen für die Lehre maßgerecht für den Dozenten erstellen und anpassen.

Aber man muss und soll vorerst nicht auf den linearen Text verzichten. Vielmehr kann reichlich Text durch eine Applikation wie TheBrain ${ }^{52}$ visuell aufbereitet werden und systematisch miteinander verknüpft werden, um den Lehrprozess, aber auch den Lernprozess zu unterstützen und zu fördern.

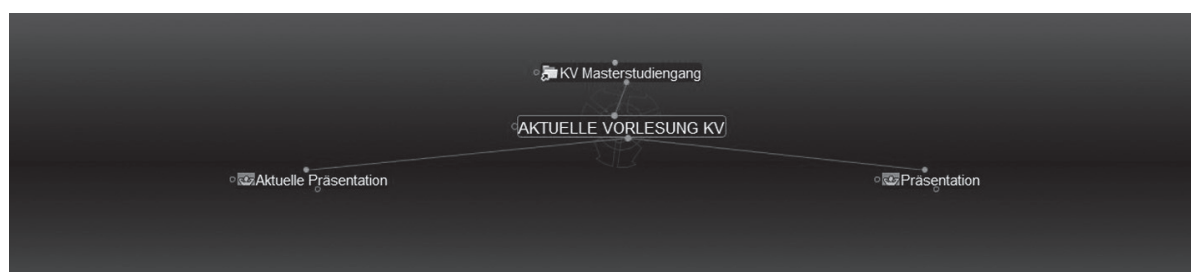

Abb. 8: Snapshot von der Applikation TheBrain, vgl. www.thebrain.com (11.3.2014)

Aber auch der Einsatz von UML ist eine visuelle Möglichkeit, Wissen besser zu strukturieren und in den Lehr- und Lernprozess zu implementieren. ${ }^{53}$

51 „Prezi ist ein plattformunabhängiges, früher ausschließlich cloud-abhängiges Präsentationsprogramm, das heute auch wie PowerPoint 'offline' verwendet werden kann. Mit der Software kann auf Basis der Flash-Technologie eine Präsentation auf einem virtuellen, unendlich großen Blatt Papier erstellt werden, auf dem man sich durch Maussteuerung bewegen sowie hinein- und heraus zoomen kann." So die kurze und prägnante Wikipediabeschreibung. Die Applikation steht Nutzern seit 2009 zur Verfügung; vgl. auch www.prezi.com. Neu hinzugekommen ist auch die App „Spacedeck“, ein Online Whiteboard und Collaboration Tool, mit guten Ansätzen, vgl. www.spacedeck.com.

52 Teile der Anwaltschaft und die Justiz (in einigen Bundesländern) verwenden bereits die Applikation „Normfall“ mit der Möglichkeit das Relationsmodul als visuelle Unterstützung einzusetzen.

53 Engesser argumentiert sehr interessant, „dass Strukturen sich oftmals hinter dem Text verbergen und sowohl Rechtsanwender wie auch Rechtsunterworfene vor Herausforderungen stellen. UML (Unified Modeling Language) kann das Recht durch eine vereinfachte Darstellung im Rahmen der Visualisierung verständlicher machen. Entwickelt wurde UML von objektorientierten Programmierern für den „Transfer“ von Realität in die Computersprachen. Mit seinen Metaebenen und Diagrammtypen ermöglicht es aber auch den „Transfer“ von Recht(srealitäten) in strukturierte und letztlich verständliche Bilder. Damit kann die Rechtsvisualisierung sich ein neues Gebiet erschließen und einen Beitrag zur einfacheren und letztlich besseren Rechtsanwendung leisten."; vgl. dazu ausführlich Engesser, Rechtsvisualisierung mittels UML am Beispiel des Verwaltungsrechts. 


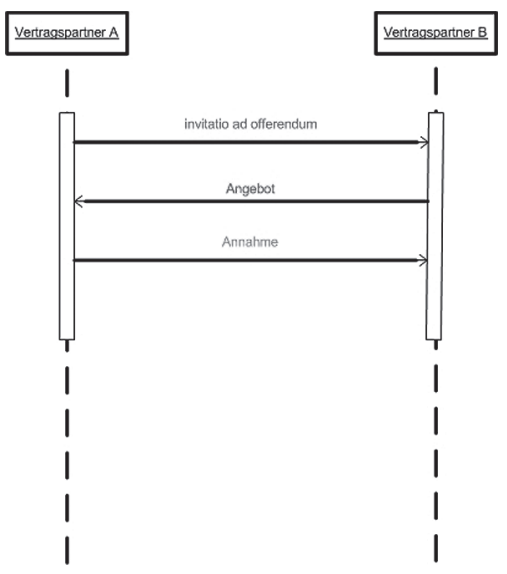

Abb. 9: Vereinfachte UML Sequenz von Aiman Khalil

\section{d. Beispiel 4: Der Einsatz von multisensorischen Hilfsmitteln in Form der audio-visuellen Unterstützung ${ }^{54}$}

Der Einsatz von Film- und Videosequenzen sowie von Trailern als Visualisierungshilfe im Lehrbetrieb ist immer noch ungewöhnlich. ${ }^{55}$

Gerade Filmsequenzen schaffen es, bei der aktuellen Generation und den nachfolgenden Generationen von Studierenden fallspezifische Besonderheiten besser darzustellen.

Die jetzigen und zukünftigen Generationen von Studierenden werden mittels dieser visualisierten Hilfsmittel Sachverhalte und rechtliche Subsumtionen leichter umsetzen als durch eine klassisch-lineare Form. Die Studierenden sind audiovisuell veranlagt. 56

Als Beispiel kann der Fall Donoghue vs. Stevenson ${ }^{57}$ angeführt werden. In Donoghue vs. Stevenson - „the case about the snail in the ginger beer in a wee cafe in Paisley” - wird sehr eindrucksvoll ein Sachverhalt dargestellt, der sich bei den Studierenden einprägt und zu reger Diskussion und praktikablen Lösungen geführt hat. Eine an-

54 Ich subsumiere die audio-visuelle Unterstützung als Teilbereich unter dem Begriff „multisensorisches Recht".

55 Erste Ansätze finden sich bei TeleJura unter http://www.telejura.de/ (20.1.2014). Erinnert sei an die Kurztrailer „Der 7. Sinn“, mit verkehrsrechtsdidaktischen Themen, die zugegebenermaßen aus heutiger Sicht sehr anachronistisch wirken, aber von der Idee und der Art des Schnitts gut gemacht waren: Beispiele kann man unter folgendem Link betrachten: http://www.youtube.com/watch?v=qpJbFWvOjlI (3.3.2014).

56 Großfeld, in: ZVglRWiss 2010, S. 379 (383), der dies „für alle Menschen“ so sieht.

57 Donoghue (or McAlister) vs. Stevenson, [1932] All ER Rep 1; [1932] AC 562; House of Lords; bei Donoghue vs. Stevenson handelt es sich um eine "tort of negligence"-Problematik des englischen Legal systems. 
schließende Besprechung mit Studierenden hat eine positive Resonanz hin zum Einsatz von audio-visuellen Material zur Folge gehabt. ${ }^{58}$

Kritik an diesem Lösungsansatz durch den Einsatz von Filmen im Hochschulalltag wird nur auf der Grundlage der Machbarkeit und Kosten erhoben. Aber gerade der Studiengang Rechtswissenschaft und der Studiengang Wirtschaftsrecht an Hochschulen für angewandte Wissenschaften sind es, die im Gegensatz zu vielen anderen Sozial- und Naturwissenschaften (MINT) vergleichsweise geringe Kosten pro Studierenden verursachen. Hier sind Mittel einzuplanen, die langfristig zu einem Filmarchiv führen, das bei herausragenden Lehrbuch-/Standardfällen wiederholt in Vorlesungen eingesetzt werden könnte.

Die Kombination von auditiven und visuellen Elementen der Aufnahme führt insbesondere für Studierende der Anfangssemester zu einem einprägsamen und bekannten Einstieg in neue Rechtsgebiete. ${ }^{59}$

Auch das Postulat der Implementierung eines bereits mehrfach angesprochenen Lehrstuhls für Rechtsvisualisierung und Rechtskommunikation würde mit einem geeigneten Mitarbeiterstamm wesentlich und analog den Rechtshistorischen Lehrstühlen und der Verwaltung deren rechtshistorischer Archive, eine visuelle und auch audio-visuelle Datenbank für die verschiedenen juristischen Fachdisziplinen gemeinsam mit den fachbezogenen Lehrstühlen und Dozenten initiieren, implementieren und verantworten. Es sei angemerkt, dass der Einsatz von audio-visuellen Kommunikationsmöglichkeiten keine Zukunftsmusik ist, vielmehr in praxi eine täglich eingesetzte Möglichkeit der Vernehmung von Zeugen. ${ }^{60}$ Der VGH Kassel hatte unlängst über eine Vernehmung einer Vertrauensperson der Polizeibehörden zu urteilen, wobei diese „an einem anderen Ort bei gleichzeitiger Übertragung von Ton und Bild in das Sitzungszimmer übertragen wurde“. ${ }^{61}$

Die Vorbehalte der Polizeibehörden gegen diese Hilfsmittel sind insoweit berechtigt, dass durch die überwiegend sehr unprofessionelle Umsetzung nicht die Sicherheit des zu schützenden Vernehmenden garantiert werden kann. Ursache hierfür ist aber nicht die „Methode selbst“, sondern die teilweise vorherrschende Art der Ausführung oder Umsetzung. Auch hier sollte wiederum durch einen Lehrstuhl für Rechtsvisualisierung, Rechtskommunikation und Rechtsdidaktik oder eine speziellen Pro-

58 Der Versuch wurde im WS 2013/2014 in einer Vorlesung zum Internationalen Recht, im 4. Semester durchgeführt.

59 Der Ginger beer-Fall ist sowohl als Film als auch in seiner Zeichentrickvariante auf http:// www.youtube.com/watch?feature=player_embedded $\& v=y L l e V 7 X h k R I \quad$ und http:// www.youtube.com/watch?v=kBlpl2c-uaU\&feature=player_embedded einsehbar (2.3.2014).

60 Vgl. exemplarisch und aktuell VGH Kassel „Unwirksame Sperrerklärung für audiovisuelle Vernehmung eines V-Mannes der Polizei“, NJW 2014, S. 240; Die Statuten und Verfahrensregeln internationaler Strafgerichte regeln dies auch schon seit Jahren ganz ausdrücklich.

61 VGH Kassel NJW 2014, S. 240. 
fessur an den Hochschulen das Fachwissen gebündelt und die neuen Erkenntnisse auch der Praxis zur Verfügung gestellt werden. ${ }^{62}$

\section{B. Conclusio}

Das Ergebnis fällt im Hinblick auf Handlungsabstinenz der wissenschaftlichen Intelligenz, des mangelnden Problembewusstseins durch die Hochschulen im Allgemeinen und der damit verknüpften Entscheidungsträger zunächst sehr kritisch aus.

Die Initiierung und Implementierung der visuellen Jurisprudenz scheitert derzeit nicht nur an den äußeren Einflüssen. Auch im Innenverhältnis ist unter den zahlreichen sogenannten Meinungsmachern derzeit eine gespenstische Ruhe eingekehrt. Diskutiert und geforscht wird ausführlich in Übersee. ${ }^{63}$ Von den deutschen Universitäten und Hochschulen hört man, speziell auf die Rechtsvisualisierung gemünzt, bis auf wenige Ausnahmen ${ }^{64}$ wenig bis gar nichts. Hier wäre der mutige Schritt zu einer jährlichen Tagung an einer deutschen Universität, zur wegweisenden Komprimierung der Meinungsvielfalt in dieser Disziplin hilfreich und der richtige Weg. Gerade die Meinungsvielfalt in der visuellen Jurisprudenz ist es, die einen aktiven Beitrag für die Lehr- und Lerndidaktik in den Rechtswissenschaften ermöglichen sollte. Sie ist eine Basis und ein Wissenspool, der für die erfolgreiche Fortführung des eingeschlagenen Weges von großer Bedeutung ist. Die Öffnung der Rechtsvisualisierung zum multisensorischen Recht bis hin zu einem möglichen Endstatus, der visuellen Jurisprudenz, mit allen denkbaren Erneuerungen, sind grundlegende Voraussetzung, um den wissenschaftlichen Fortschritt zu garantieren und den Rechtswissenschaften Unterstützungsleistungen zu bieten. Dazu bedarf es der Etablierung eines speziellen Lehrstuhls bzw. der Bereitstellung einer Professur an den Hochschulen für angewandte Wissenschaften. Einem zukünftigen Lehrstuhl/einer Professur obliegt die Steuerungsoptimierung für den gesamten Fachbereich/der Fakultät.

Dies kann nur durch Einbindung der anderen juristischen Fachdisziplinen wirksam erfolgen. Dazu bedarf es der wissenschaftlichen Akzeptanz durch alle, die Recht lehren. Die Praxis und die Justiz muss zeitnah eingebunden werden. Daraus resultiert die Implementierung einer neuen „Prüfungsmatrix“. Dies bedeutet dass neue, auch computerunterstützte Hilfsmittel Zugang in den Klausuren und Prüfungsalltag finden werden. ${ }^{65}$

62 Dann können mittels der Rechtsvisualisierung als neue Disziplin auch die modernen Grundsätze eines Fair Trials im Strafprozess für alle beteiligten effizient eingehalten und durchgesetzt werden.

63 Lediglich an der Uni Zürich (nur englischsprachig) und beim Internationalen Rechtsinformatiker Symposium in Salzburg (initiiert von Schweighofer u.a.) sind Plattformen zum Austausch möglich.

64 Exemplarisch die Jahrestagungen des ZeRF in Hamburg sowie im Zweijahresrhythmus das Institut für Rechtsdidaktik in Passau.

65 Referendarklausuren im Studium enthalten erläuternde und farblich beschreibende Hinweise zum Klausursachverhalt. Assessorklausuren bestehen sowohl aus der digitalen Papierakte als auch aus einer oder mehreren Videosequenzen, die es den Prüfern ermöglicht, auch Zeugenaussagen, Verkehrsunfälle und andere visuell darstellbare Sachvorträge mittels Tablet und Kopfhörer zur Verfügung zu stellen. 
Der Weg in die Visuelle Jurisprudenz ist trotz der anfänglichen Kritik auch mit den heutigen (auch technischen) Lösungsmöglichkeiten machbarer und effektiver zu gestalten, als das bisherige jahrhundertalte Lernsystem, welches schon in vergangenen Digestenzeiten praktiziert wurde.

Die Rechtswissenschaft darf in ihrer Isolierung als Wissenschaft nicht verharren, sondern muss sich interdisziplinär anderen Bereichen öffnen, um eine zeitgemäße Didaktik zu verfolgen, mit der den Ansprüchen der heutigen Studierenden Rechnung getragen wird. Eine zeitgemäße juristische Didaktik muss interdisziplinär mit anderen Wissenschaften verstärkt zusammenarbeiten, um der gegenwärtigen und zukünftigen Klientel an Studierenden gerecht zu werden. Der Paradigmenwechsel hat bereits begonnen.

\section{Literaturverzeichnis}

Bengez, Rainhard Z., „Das Recht war und ist ein normatives Praxissystem, das in den letzten Jahrhunderten textuell überformt wurde um sich nunmehr in ein maschinendominiertes Herrschaftssystem zumindest partiell zu wandeln.“, http://rechtundlogik2012.quantius.org/ (9.1.2014).

Bredekamp, Horst, Die Eigenkraft der Bilder, in: Hohe Luft, Ausgabe 2/2012 S. 68-72.

Brunschwig, Colette, Multisensory Law and Legal Informatics - A Comparison of How these Legal Disciplines Relate to Visual Law, in: Geist et al. (Hrsg.), Structuring Legal Semantics, Festschrift für Erich Schweighofer, Bern 2011, S. 573-667.

Buzan, Tony/Buzan, Barry, Das Mind-Map Buch, München 1996.

Ebster, Claus/Stalzer, Lieselotte, Wissenschaftliches Arbeiten für Wirtschafts- und Sozialwissenschaftler, 2. Auflage, Stuttgart 2003.

Eco, Umberto, Wie man eine wissenschaftliche Abschlussarbeit schreibt, 10. Auflage, Stuttgart 2003.

Engesser, Michael, Rechtsvisualisierung mittels UML am Beispiel des Verwaltungsrechts, Innsbruck 2012. Gergen, Kenneth J., An Invitation to Social Construction, Thousand Oaks 1999.

ders,. Konstruierte Wirklichkeiten - Eine Hinführung zum sozialen Konstruktionismus, Stuttgart 2002.

ders./Gergen, Mary, Einführung in den sozialen Konstruktionsimus, Heidelberg 2009.

Gerhards, Gerhard, Seminar-, Diplom und Doktorarbeit, 8. Auflage, Stuttgart 1995.

Giersberg, Georg, Steuererklärung in der Datenwolke, in: FAZ vom 28.2.2014, S. 21.

Gross, Thomas, Besprechung Westerkamp, in: FAZ vom 15.5.2013, S. N3.

Großfeld, Bernhard, Musik und Recht, in: ZVglRWiss 2010, S. 379-397.

ders. Zeichen und Bilder im Recht, in: NJW 1994, S. 1911-1916.

Haapio, Helena/Passera, Stefania, „Visual Law: What Lawyers Need to Learn from Information Designers“, http://blog.law.cornell.edu/voxpop/2013/05/15/ visual-law-what-lawyers-need-to-learn-frominformation-designers/ (19.1.2014) oder Beispiele auf den Seiten von http://www.visuelle-jurisprudenz.de (19.1.2014).

Heindl, Pete/Kahlig, Wolfgang/Österreicher, Theodor/Sommer, Andreas, WGG II strukturiert, Wien 2012.

Herzog, Felix, Strafrecht illustrated, 30 Fälle aus dem Strafrecht in Wort und Bild, Hamburg 2007.

Hilgendorf, Eric (Hrsg.), Beiträge zur Rechtsvisualisierung, Berlin 2005.

Holzer, Florian, Rechtsvisualisierung im Strafrecht, Ein Beitrag zur Steuerung der visuellen Rechtskommunikation, unveröff. Diss., Würzburg 2011. 
Karmasin, Matthias/Ribing, Rainer, Die Gestaltung wissenschaftlicher Arbeiten: Ein Leitfaden für Seminararbeiten, Bachelor-, Master- und Magisterarbeiten sowie Dissertationen, 7. Auflage, Stuttgart 2012.

Kertai, Benjamin, Das Bild im Strafverfahren - Strafprozessuale Probleme bei der Visualisierung, in: MMR 2011, S. $716 \mathrm{ff}$.

Khalil, Aiman, Rechtsvisualisierung durch Mindmaps - (k)eine Modeerscheinung, in: Schweighofer/Geist/ Heindl (Hrsg.), 10 Jahre IRIS: Bilanz und Ausblick. Tagungsband des 10. internationalen Rechtsinformatik Symposions IRIS 2007, Stuttgart 2007, S. 468-473.

ders., Visuelle Jurisprudenz, Würzburg 2011.

ders., Neue Zeichen und neue Bilder im Recht - von Leibniz bis Lachmayer, in: Schweighofer et al. (Hrsg.), Festschrift für Friedrich Lachmayer, Zürich 2014, S. 965-975.

Lachmayer, Friedrich, Graphische Darstellungen im Rechtsunterricht, in: Zeitschrift für Verkehrsrecht, 1976, S. 230-234.

ders., Graphische Darstellungen als Hilfsmittel des Gesetzgebers, in: Klug/Ramm/Rittner/Schmiedel (Hrsg.), Gesetzgebungstheorie, Juristische Logik, Zivil- und Prozessrecht, Gedächtnisschrift für Jürgen Roedig, Berlin u.a. 1978, S. 50-58.

ders./Hoffmann, Harald/Čyras, Vytautas, Multisensorische Modelle des Rechts, in: Jusletter IT (Editions Weblaw, Bern) ISSN 1664-848X 06/2012.

Mielke, Bettina/Wolff, Christian, Welche Farbe hat das Recht?, in: Schweighöfer et al. (Hrsg.), Semantisches Web und Soziales Web im Recht, Tagungsband des 12. Internationalen Rechtsinformatik-Symposions IRIS 2009, Wien 2009, S. 301-308.

Putzke, Holm, Juristische Arbeiten erfolgreich schreiben: Klausuren, Hausarbeiten, Seminare, Bachelorund Masterarbeiten, 4. Auflage, München 2012.

Röhl, Klaus/Ulbrich, Stefan, Recht anschaulich - Visualisierung in der Juristenausbildung, Köln 2007.

Sachs-Hombach, Klaus, Das Bild als kommunikatives Medium, Elemente einer allgemeinen Bildwissenschaft, 2. Auflage, Köln 2006.

Schimmel, Roland/Weinert, Mirko/Basak, Denis, Juristische Themenarbeiten: Anleitung für Klausur und Hausarbeit im Schwerpunktbereich, Seminararbeit, Bachelor- und Master-Thesis (Schwerpunkte Klausurenkurs), 2. Auflage, München 2011.

Schindler, Steffen, Aufstiegsangst? - Eine Studie zur sozialen Ungleichheit beim Hochschulzugang im historischen Zeitverlauf, mit einem Kommentar von Walter Müller, herausgegeben von der Vodafone Stiftung Deutschland, Düsseldorf 2012, als pdf erhältlich unter vodafone-stiftung.de (29.1.2014).

Silvestrini, Narciso/Fischer, Ernst Peter, Farbsysteme in Kunst und Wissenschaft, Stromer (Hrsg.), 3. Auflage, Köln 2005.

Stalzer, Claus/Stalzer, Liselotte, Wissenschaftliches Arbeiten für Wirtschafts- und Sozialwissenschaftler; 4. Auflage, Wien 2013.

Thüsing, Gregor, Bilder des Rechts, in: NVwZ 2013, S. 193-195.

Volk, Klaus/Holzer, Florian/Heller, Simon, Panorama Strafrecht, Übersicht zum Allgemeinen Teil, München 2007.

Wander, Karl Friedrich Wilhelm, Deutsches Sprichwörter-Lexikon. Ein Hausschatz für das deutsche Volk, Augsburg 1987.

Westerkamp, Dirk, „Der dramatische Moment. Fünf Reflexionen über ikonische Prägnanz“, in: Zeitschrift für Ästhetik und Allgemeine Kunstwissenschaft, 58 (2013), S. 35-55.

Winter, Rainer, Sozialer Konstruktionismus, in: Mey/Mruck (Hrsg.), Handbuch Qualitative Forschung in der Psychologie, Heidelberg 2010, S. 123-135. 